\title{
¿POR QUÉ HABLAR DE PSICOLOGÍA DE LA EMERGENCIA EN AMÉRICA LATINA? UNA DISCUSIÓN NECESARIA
}

Eduardo Sandoval-Obando ${ }^{1, *}$

\section{INTRODUCCIÓN}

A propósito de las complejidades socio-naturales, urbanísticas, ambientales, climatológicas e incluso geográficas que caracterizan el continente americano, y particularmente a Chile, emergen una serie de condiciones de riesgo y amenazas que colocan en jaque y en permanente cuestionamiento, las políticas públicas en lo relacionado a la gestión del riesgo y reducción de desastres. Más aún, existen antecedentes recientes de eventos socio-naturales que han afectado significativamente a las personas, tales como: el terremoto del 27 de Febrero 2010; la erupción del volcán Villarrica y Calbuco durante el año 2015; los mega incendios forestales durante el 2017; el aluvión en Villa Santa Lucía ocurrido en diciembre 2017 y el aumento de la actividad sísmica asociada al Complejo Volcánico Nevados de Chillán, entre otros.

Estas condiciones develan una problemática relevante y actual en Chile y el mundo, reafirmando la necesaria discusión en torno al aporte y validación de la Psicología de la Emergencia, como un ámbito del saber, especializado en responder al tratamiento de personas y/o comunidades que experimentan el trauma, antes, durante y tras la presentación de un evento que altera significativamente su calidad de vida, así como el normal funcionamiento de los servicios básicos presentes en los territorios afectados.

Precisamente por ello, resulta necesario delimitar ámbitos de acción y alternativas posibles de mejora, en torno a la comprensión de los fenómenos ya descritos, describiendo las consecuencias directas o indirectas que provocan estos eventos sobre la vida de las personas y sus comunidades de origen, tensionando el marco institucional vigente en Chile en lo relacionado a la Gestión del Riesgo de Desastres (GRD), de acuerdo a la frecuencia, magnitud o gravedad con que se presenta un determinado evento.

En complemento a lo anterior, se plantean un conjunto de reflexiones en torno a esta disciplina y su importancia en la preparación de los equipos de intervinientes que participaron durante emergencias ocurridas durante los últimos años en la Región de Los Ríos, Chile. De esta manera, este artículo se construye a partir de la práctica situada y experiencia profesional acumulada por el autor, tanto en el ejercicio de la docencia universitaria para formar a futuros psicólogos, como desde los procesos de intervención clínica (en contextos de encierro y Atención Primaria en Salud Mental), así como en lo relacionado a diversos instancias de formación y capacitación destinada a funcionarios/as pertenecientes a las Fuerzas Armadas y de Orden (Ejército de Chile y Carabineros respectivamente). El análisis reflexivo de las problemáticas descritas y vividas por las personas y las comunidades afectadas por estos eventos, pero particularmente las experiencias aportadas por los intervinientes en el marco de los diferentes procesos de contención e intervención en crisis, obtenidos en el trabajo académico y profesional, se transforman en el componente central que fundamenta estas reflexiones, avanzando en la comprensión dinámica, actualizada y local de los complejos procesos de subjetivación y construcción de saberes desplegados por los profesionales de apoyo sanitario y psicosocial que asumen responsabilidades y tareas específicas tras la ocurrencia de estos eventos.

Paralelamente, la discusión que se plantea adquiere especial interés en la medida que sea capaz de cultivar nuevos espacios de discusión, análisis y sistematización en el ámbito universitario
1. Escuela de Psicología, Facultad de Ciencias Sociales y Humanidades, Universidad Autónoma de Chile, Temuco, Chile.

*Autor de correspondencia: eduardo.sandoval.o@gmail. com

\section{RECIBIDO}

13 de septiembre de 2018

\section{PUBLICADO}

1 de enero de 2019

\section{Formato cita}

Recomendada (APA):

Sandoval-Obando, E. (2019). ¿Por qué Hablar de Psicología de la Emergencia en América Latina? Una

Discusión Necesaria, Revista de Estudios

Latinoamericanos sobre Reducción del Riesgo de Desastres REDER, 3(1), pp.102-107.

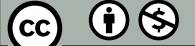

Todos los artículos publicados en REDER siguen una política de Acceso Abierto y se respaldan en una Licencia CreativeCommons Atribución-NoComercial 4.0 Internacional.

\section{Revista de Estudios}

Latinoamericanos sobre

Reducción del Riesgo de

Desastres (REDER)

Diseño: Lupe Bezzina Tipografía: Hospital 
y la sociedad en general, respecto a los desafíos que enfrentan los países en materia de prevención y GRD, transfiriendo en definitiva, elementos técnicos y conceptuales, que enriquezcan investigativamente a las ciencias sociales y humanas, dado que una GRD eficaz es una inversión necesaria para avanzar un desarrollo sostenible y armónico con el territorio.

\section{DESARROLLO}

\section{Psicología de la Emergencia: Ámbitos de acción e intervención}

La Psicología de la Emergencia es un campo del saber especializado en el estudio del comportamiento humano ante, durante y tras la presentación de eventos o situaciones de emergencia. Al respecto, Barrales, Marín y Molina (2013) relacionan estos momentos con las etapas del ciclo metodológico de un desastre en la perspectiva de la gestión del riesgo: prevención, reacción, emergencia y reconstrucción. Otros autores, caracterizan la Psicología de la Emergencia como un área de la psicología abocada al:

"Estudio de las reacciones de los individuos y de los grupos humanos en el antes, durante y después de una situación de emergencia o desastre, así como de la implementación de estrategias de intervención psicosocial orientadas a la mitigación y preparación de la población, estudiando cómo responden los seres humanos ante las alarmas y cómo optimizar la alerta, evitando y reduciendo las respuestas inadaptativas durante el impacto del evento y facilitando la posterior rehabilitación y reconstrucción".

(Valero, 2002)

De acuerdo a lo anterior, y en base a la revisión realizada (Araya, 1992; Valero et al., 2007; Parada, 2008), se podría señalar que los ámbitos de estudio e intervención para la Psicología de la Emergencia serían:

- Comprender los procesos psicológicos básicos y complejos, que emergen durante la presentación de una emergencia o desastre.

- Desarrollar y desplegar técnicas psicológicas para situaciones de emergencia, tales como los Primeros Auxilios Psicológicos, la Intervención en Crisis, Gestión de la Espera, Protocolos de Desactivación en intervinientes, entre otros.

- Apoyar los procesos de selección de personal para integrar los equipos de intervinientes que se despliegan durante la presentación de una catástrofe: Comités Operativos de Emergencia (COE); bomberos, equipos de salud; rescatistas, integrantes de las Fuerzas Armadas y de Orden, entre otros.

- Promover instancias de capacitación, psicoeducación y formación continua en temáticas vinculadas al afrontamiento de emergencias, cultura de la prevención y comprensión de protocolos que regulan los fenómenos ya señalados.

\section{Primeros Auxilios Psicológicos: Un enfoque de intervención}

Los Primeros Auxilios Psicológicos (PAP) han cobrado importancia a partir de los diferentes eventos, conflictos y desastres ocurridos en Chile y América Latina. Según lo señalado por algunos autores (Fernández, 2005; Gantiva, 2010; Salas, 2012; Cortés \& Figueroa, 2018), los PAP son un enfoque clínico de intervención modular, basado en evidencias y destinado al apoyo y contención de todas aquellas personas pertenecientes a una determinada comunidad o región que se ha visto afectadas por un incidente crítico: ej. un terremoto, conflicto armado, o tsunami. De esta manera, se han transformado en la primera respuesta que reciben los afectados, y por ello, generalmente se aplican dentro de las primeras 72 horas de ocurrida la emergencia o catástrofe (Martín \& Muñoz, 2009).

Forman parte de la primera fase de la intervención en crisis, adquiriendo sentido de aplicación en aquellos casos en que los mecanismos de adaptación y afrontamiento de los sujetos se encuentran desbordados, experimentando un alto nivel de dificultad para retomar la cotidianidad de su vida. Esta situación provoca en los afectados un estado de malestar, ambivalencia, impulsividad y excitación, que afecta notoriamente su capacidad para tomar decisiones tras la presentación del evento. Por ello, la aplicación de esta técnica persigue 3 grandes propósitos:

- Reducir el nivel de estrés inicial provocado por la presentación del evento traumático, reduciendo la sintomatología somática vinculada al trauma vivido. 
- Fomentar la adaptación de la persona y/o colectivo afectado al corto, mediano y largo plazo. Con esto se persigue disminuir la probabilidad de aparición de psicopatologías que afecten al sujeto o colectivo afectado.

- Potenciar el despliegue de estrategias de afrontamiento activas frente al evento traumático, posibilitando el desarrollo de la resiliencia, el fortalecimiento de los lazos de asociatividad, la reestructuración cognitiva y la paulatina organización de las personas y/o colectivo afectado.

\section{Formación y educación en torno a la Psicología de la Emergencia: Reflexiones profesionales desde una perspectiva local}

La especialización y formación en Psicología de la Emergencia es un campo emergente y en pleno desarrollo. Sin embargo, ha adquirido protagonismo e importancia como consecuencia de una larga historia de emergencias y desastres socio-naturales que Chile ha enfrentado, producto en parte, por las características geográficas, históricas, socioculturales y ambientales que caracterizan y complejizan el territorio nacional, reflejando un alto grado de vulnerabilidad de la población frente a estos eventos.

Lo cierto es que el quehacer profesional del autor en estas temáticas adquiere relevancia y pertinencia técnica, a partir de las diversas instancias de formación y capacitación en psicología de la emergencia, educación en salud mental y actualización en protocolos de desactivación para intervinientes que ha realizado durante los últimos años en su rol de Psicólogo e Interventor clínico para personal adscrito a las Fuerzas Armadas y de Orden, las que en su mayoría se han realizado de manera voluntaria en Valdivia (Región de Los Ríos, Chile). El propósito de estas acciones es transferir herramientas técnicas que contribuyan a la construcción y promoción de una cultura de la prevención en la sociedad, y particularmente, la de promover la salud mental positiva en los equipos de intervinientes que se despliegan en ambientes altamente estresantes y caóticos.

A partir de la experiencia acumulada por el autor, es posible señalar algunos ejes prioritarios de acción que serían susceptibles de incorporar, en la comprensión y abordaje integral de estas problemáticas, tales como:

- Emergencias y desastres socio-naturales como fenómenos complejos: Se observa la necesidad de educar y sensibilizar a los intervinientes (equipos de salud, apoyo psicosocial, personal adscrito a las Fuerzas Armadas y de Orden) en la noción de que estos eventos tienen un impacto al corto, mediano y largo plazo en la vida de las personas y comunidades afectadas. Por ello, se sugiere una correcta valoración, interpretación y sistematización de los factores psicosociales, políticos, culturales y relacionales que condicionan la capacidad de respuesta de los afectados. Estos aspectos son clave en la fase de reconstrucción y rehabilitación, en donde adquiere importancia el apoyo social y las redes comunitarias para resignificar el impacto de una catástrofe en la población (particularmente, cuando existen pérdidas de vidas humanas).

- Incorporación de profesionales expertos en salud mental dentro de los equipos de intervinientes frente a situaciones de emergencia o desastres: Desde luego que nuestro país ha ido dando pasos importantes en mejorar su capacidad de respuesta frente a estos eventos. Sin embargo, resulta prioritario que se trabaje sistemática y transversalmente en la incorporación de psicólogos y psicólogas con formación en emergencias y manejo del trauma psíquico, dentro de los equipos socio-sanitarios (SAMU, Unidades de Salud Mental que colaboren activamente con FF.AA y de Orden por ejemplo), para el soporte, recuperación y rehabilitación de la población afectada por una catástrofe.

- La Cultura de la Prevención en la ciudadanía: Intervenir oportuna y correctamente frente a las emergencias y desastres socio-naturales que puedan presentarse en Chile no es una responsabilidad única de los equipos de primera respuesta o Fuerzas Armadas y de Orden. Al respecto, se cree necesario continuar promoviendo una institucionalidad moderna y proactiva que trabaje decididamente en la generación de políticas de prevención que eviten o reduzcan al máximo las pérdidas humanas, mitigando en parte, el daño de los afectados (Chan et al., 2009).

- Respeto y valoración por el saber local: Uno de los puntos más valiosos detectados en zonas de catástrofes, radica en que los intervinientes sean capaces de valorar el 
conocimiento popular de las comunidades, haciéndolos partícipes de los planes de prevención y protección ante desastres socio-naturales. De esta manera, es posible articular políticas de gestión del riesgo de desastres con un enfoque participativo, intersectorial y coherente con las necesidades verdaderamente situadas en el territorio.

\section{Emergencias y desastres: Implicancias para la salud mental}

El estudio y comprensión de las consecuencias psicológicas que emergen tras la presentación de un desastre socio-natural, ha posibilitado avanzar en la revisión y sistematización del impacto generado sobre la salud mental de las personas o comunidades que afrontan estos fenómenos, vale decir, la presencia de síntomas o trastornos (Cova \& Rincón, 2010). Desde esta perspectiva, se ha observado la presencia de sintomatología ansiosa y psicosomática inespecífica relacionada con el estrés como una de las consecuencias más consistentemente observadas (Kuwabara et al., 2008), la aparición de trastornos del estado de ánimo (Van Griensven et al., 2006) y la presencia del Trastorno por Estrés Postraumático, como una de las patologías más estudiadas y revisadas tras estos eventos (Neria et al., 2008). En otra esfera, se ha monitoreado y otorgado mayor importancia a los procesos de reconstrucción y rehabilitación, en donde adquieren notoriedad otras problemáticas psicosociales, relacionadas con los procesos de duelo, las pérdidas económicas y laborales, la relocalización y la pérdida del hogar, entre otros.

La revisión realizada permite destacar que existe consenso nacional e internacional respecto a las múltiples consecuencias psicológicas y sociales que acarrea una emergencia o desastre socio-natural, alterando notoriamente la vida de las personas y sus comunidades (Pérez, 2012).

No obstante a lo anterior, y más allá de la perspectiva clínica, el desafío para los países radica en continuar mejorando sus protocolos y mecanismos de respuesta disponibles para afrontar eventos de alta magnitud y severidad que superan muchas veces, los recursos existentes en el territorio. De igual manera, adquiere importancia el apoyo social percibido y las redes comunitarias activas, como dinámicas relacionales y socio-culturales que ayudarían al proceso de resignificación del impacto de una catástrofe en la población (particularmente, cuando existen pérdidas de vidas humanas), convirtiéndose en tópicos de especial interés para la investigación aplicada, integrando aspectos como calidad de vida, cuidado y buen trato en la infancia, protección integral de la salud (física y mental), participación ciudadana y la tan anhelada cultura de la prevención frente a emergencias o desastres socio-naturales en América Latina.

\section{COMENTARIOS FINALES}

A modo de conclusión, estas reflexiones pretenden enriquecer la discusión acerca de los desafíos existentes en torno a la construcción de una cultura de la prevención frente a las emergencias y desastres socio-naturales en América Latina (Sandoval, 2016). Al respecto, y a propósito de lo ampliamente descrito en el Marco de Sendai para la Reducción del Riesgo de Desastres 2015-2030 (ONU, 2015), resulta necesario que los países puedan centrarse tanto en las amenazas naturales como de origen humano, tomando conciencia de los riesgos ambientales, tecnológicos y biológicos conexos. Precisamente por ello, este trabajo promueve la resiliencia sanitaria y socio-comunitaria, reconociendo que la Reducción del Riesgo de Desastres (RRD) es una inversión rentable en la prevención de pérdidas futuras, favoreciendo el desarrollo sostenible de los países.

Al mismo tiempo, y de acuerdo a la experiencia acumulada, se propone avanzar concretamente en cuatro ámbitos:

- Marco urbano-legal: Mejoramiento de las políticas arquitéctonicas; reconstrucción de viviendas con estándar antisísmico; reubicación de aquellos asentamientos urbanos en zonas geográficas de riesgo; diseño del plan urbano en sintonía y coherencia a las características socio-naturales presentes en el territorio; accesos inclusivos y vías de evacuación claras, efectivas y que cumplan con estándares internacionales para grupos con características diversas (adultos mayores, personas no videntes, discapacidad motora, entre otros).

- Medidas socio-educativas: Planes y programas educativos que aborden esta temática en los diferentes ámbitos del saber; capacitación y educación continua en torno a la prevención; entre otros.

- Medidas sanitarias en emergencia: Mejoramiento de las coordinaciones entre dispositivos de emergencia; fortalecimiento de los procesos e instancias de capacitación y 
orientación en salud mental a los integrantes de los equipos de primera respuesta e intervinientes; monitoreo sistemático de las eventuales reacciones o cambios comportamentales que exhiba el personal desplegado en zonas de catástrofes, durante e inmediatamente después de un incidente crítico, así como también en períodos no críticos; difusión y entrenamiento en torno a los protocolos de desactivación para intervinientes; psicoeducación en torno a la psicología de la emergencias y gestión del riesgo de desastres, entre otras.

- Medidas políticas: Articulación de una institucionalidad moderna y eficiente en torno al abordaje de estos eventos; cooperación y transferencia de prácticas exitosas, tal como ha sido la relación con Japón; planificación estratégica en torno a la GRD; sistematización de lecciones aprendidas en torno a las catástrofes vividas como país, entre otras.

Finalmente, las reflexiones planteadas proponen también la construcción de una Política Integral de Prevención de Emergencias y Desastres Socio-naturales, diseñadas de manera participativa y con un carácter intersectorial, integrando activamente los diferentes ámbitos de la sociedad (Estado, Sociedad Civil, Academia, Privados, entre otros), posibilitando la sistematización de experiencias locales, metodologías o tecnologías exitosas en esta materia, que puedan ser transferibles a otros entornos. Sin duda alguna, América Latina posee una enorme riqueza histórica y sociocultural en lo relacionado a la prevención de estos fenómenos, y desde allí, deberíamos ser capaces de avanzar en la construcción de una verdadera cultura de la prevención para todos y todas.

\section{REFERENCIAS}

Araya, C. (1992). Psicoprevención y Psicología de la Emergencia. Santiago de Chile: Kartel.

Barrales, C., Marín, H. \& Molina, R. (2016). Estado del Arte de la Psicología en Emergencias y Desastres en Chile y América Latina. Liminales. Escritos sobre psicología y sociedad, 1(3), 123-142.

Chan, E. Gao, Y. \& Griffiths, S. (2009). Literature review of health impact post-earthquakes in China 1906-2007. Journal of Public Health, 32, 52-61.

Cortes, P. \& Figueroa, R. (2018). Manual ABCDE para la Aplicación de Primeros Auxilios Psicológicos: En Crisis Individuales y Colectivas. Santiago de Chile: PUC / CIGIDEN.

Cova, F. \& Rincón, P. (2010). El Terremoto y Tsunami del 27-F y sus Efectos en la Salud Mental. Terapia Psicológica, 28(2), 179-185.

Fernández, J. (2005). Apoyo Psicológico en Situaciones de Emergencia. Madrid: Pirámide.

Gantiva, C. (2010). Intervención en Crisis: Una Estrategia Clínica Necesaria y Relevante en Colombia. Psychologia: Avances de la Disciplina, 4(2), 143-145.

Kuwabara, H., Shiori, T., Toyabe, S., Kawamura, T., Koizumi, M., Ito-Sawamura, M., Akazawa, K. \& Someya, T. (2008). Factor impacting on psychological distress and recovery after the 2004 NiigataChuetsu earthquake, Japan: Community-based study. Psychiatry and Clinical Neurosciencies, 62, 503-507

Martín, L. \& Muñoz, M. (2009). Primeros Auxilios Psicológicos. Madrid: Síntesis.

Neria, Y., Nandi, A. \& Galea, S. (2008). Post-traumatic stress disorder following disasters: a systematic review. Psychological Medicine, 38, 467-48o.

Organización de Naciones Unidas ONU. (2015). Marco de Sendai para la Reducción del Riesgo de Desastres (2015-2030). Sendai: ONU. Recuperado de: https://www.unisdr.org/files/43291_ spanishsendaiframeworkfordisasterri.pdf [visitado el 20 de ene. de 2019]

Parada, E. (2008). Psicología y Emergencias: Habilidades Psicológicas en las Profesiones de Socorro y Emergencia. Bilbao: Desclée De Brouwer.

Pérez, P. (2012). Atención a los Problemas de Salud Mental en Situación de Catástrofes y Emergencias. En M. Desviat y A. Pérez, Acciones de salud mental en la comunidad. Madrid: Asociación Española de Neuropsiquiatría, pp. 659-671.

Salas, G. (2012). Psicología de la Emergencia y Desastres: Experiencias de Intervención en Situaciones de Crisis. Santiago de Chile: COR.

Sandoval, E. (2016, diciembre 28). La Cultura de la Prevención ante Desastres Socio-naturales: Todos Contamos. Sur Actual [online]. Recuperado de: http://www.suractual.cl/2016/12/28/la-cultura-dela-prevencion-ante-desastres-socio-naturales-todos-contamos-por-eduardo-sandoval [visitado el 20 de ene. de 2019] 
Valero, A., Gil, J. \& García, M. (2007). Profesionales de la Psicología ante el Desastre. Castelló de la Plana: Universitat Jaume I.

Valero A. Santiago. (2002). Psicología en Emergencias y Desastres. Lima: San Marcos.

Van Griensven, F., Somchai, M., Thienkrua, W., Pengjuntr, W., Lopes, B., Tantipiwatanaskul, P., Mock, P., Ekassawin, S., Varangrat, A., Gotway, C., Sabin, M., \& Tappero, J. (2006). Mental health Problems among adults in Tsunami-Affected areas in Southern Thailand. Journal of the American Medical Association, 296, 537-548. 